\title{
Seagrass beds as the buffer zone for fish biodiversity in coastal water of Bontang City, East Kalimantan, Indonesia
}

\author{
ADITYA IRAWAN ${ }^{1,2, \boldsymbol{v}}$, SUPRIHARYONO ${ }^{1}$, JOHANNES HUTABARAT $^{1}$, AMBARIYANTO $^{1}$ \\ ${ }^{1}$ Doctoral Program in Coastal Resources Management, Faculty of Fisheries and Marine Science, Universitas Diponegoro. Tembalang, Semarang 50275, \\ Central Java, Indonesia \\ ${ }^{2}$ Faculty of Fisheries and Marine Science, Universitas Mulawarman. Jl. Gunung Tabur, Gunung Kelua, Samarinda 75123, East Kalimantan, Indonesia. \\ Tel./fax.: +62-541-748648, ”email: aditya.irawan@ffpik.unmul.ac.id
}

Manuscript received: 24 November 2017. Revision accepted: 15 May 2018.

\begin{abstract}
Irawan A, Supriharyono, Hutabarat J, Ambariyanto. 2018. Seagrass beds as the buffer zone for fish biodiversity in coastal water of Bontang City, East Kalimantan, Indonesia. Biodiversitas 19: 1044-1053. The purpose of this study was to demonstrate the importance of seagrass beds as a buffer zone for fish biodiversity in a coastal ecosystem. The sampling of seagrass and fish were done in seagrass beds associated with mangrove (ST1) and coral reef (ST2). Sampling period was executed from May 2011 to May 2017. Sampling was done on the lowest tide with a six-month interval. Seagrass sampling was carried out using line transect, and fish sampling was performed using set net (belat local name) operated by local fisherman. The results showed that seagrass beds at ST1 composed of $C$. rotundata and $E$. acoroides and that at ST2 composed of $C$. rotundata, E. acoroides, $H$. pinifolia, $H$. minor and $T$. hemprichii. The total number of individual was 42574 divided into 112 fish species. There were 17 species (population: 15.18\%) associated explicitly with ST1 and 39 species (population: 34.82\%) specifically associated with ST2 while 56 fish species (population: $50.0 \%$ ) were prevalently spread in both stations. This research proved the existence of seagrass beds as a buffer zone for fish biodiversity. $\square$
\end{abstract}

Keyword: Biodiversity, connectivity, fish, seagrass beds

\section{INTRODUCTION}

Seagrass is a macrophyte in coastal water (Billah et al. 2016). Structurally, the construction of seagrass beds provides nursery grounds for fish juvenile (Jones 2014), and coral fishes (Verweij et al. 2008). It also serves as feeding grounds (Coles et al. 1993; Erftemeijer and Allen 1993; Carroll and Peterson 2013) for economically highvalue fishes (Hantanirina and Benbow 2013; Blandon and zu Ermgassen 2014), to provide food, food chain and biodiversity, and ecosystem service (Cucio et al. 2016) in supporting the coastal water productivity (Greening and Janicki 2006; Nadiarti et al. 2012; Bahlmann et al. 2015). Seagrass tends to be found close to mangrove forests and coral reefs (Short et al. 2011). This illustrates the importance of interaction in the coastal ecosystem (Hirst et al. 2016). Natural interactions within the coastal ecosystem protect the population's habitat (Unsworth et al. 2007). A combination of two habitats shows that mangroves specifically protects the coast and coral reef that forms a fringing reef for seagrass beds, which serve to control degradation of coral reefs in the long-term (Guannel et al. 2016).

By understanding the importance of the role of seagrass beds, we should have been able to manage its sustainability, but in reality, the fact shows that the expanse of seagrass beds have decreased in many parts of the world (Hughes et al. 2009; Jones 2014) and seagrass beds have been globally threatened by anthropogenic factors and climate change (Rasheed and Unsworth 2011; Grech et al.
2012; Unsworth et al. 2012; Duarte et al. 2013; Shafer et al. 2014; Mazarrasa et al. 2015). This same thing happened in the coastal area in Bontang where the expanse of seagrass beds had also been decreased as the result of the damage covering around 321.3 ha area or as much as $45 \%$ of total seagrass beds area of 714 ha (DPKP Kota Bontang 2011). Despite the fact that the composition of seagrass beds (consisting of 9 species of total 12 species found in Indonesia) (Tomascik et al. 1997; P3IK/P3O-LIPI 2000; DPKP Bontang 2015) had been known, it is a mangrove forest. Coral reef has become the priority in conservation management, and the critical role of seagrass beds in supporting fishery resources and the productivity in Bontang coastal water tends to be neglected.

This study aimed to demonstrate the importance of seagrass beds as the buffer zone for fish species biodiversity in coastal ecosystem especially the association between seagrass beds, mangrove, and coral reef, to initiate an integrated and continuous management system in the coastal ecosystem. This study focused on fish species composition and the distribution of fish length size and its interaction with species characteristic, the density of seagrass stand and the association between seagrass beds and mangrove and coral reef in Bontang coastal water. Mangrove forests and coral reef are creating the importance of interaction between coastal ecosystems. The natural interaction of coastal ecosystem provides protection for population habitat or combination of two habitats which shows that mangrove specifically provides protection to the coast and coral reef that forms a fringing reef for seagrass 
beds, and seagrass beds serve to control degradation of coral reef in a long-term.

\section{MATERIALS AND METHODS}

\section{Study area}

This research was carried out in coastal water of northern Bontang City, East Kalimantan, Indonesia. Observation station consisted of seagrass beds associated with mangrove (ST1) and seagrass beds associated with the coral reef (ST2) (Figure 1). Seagrass beds in ST1 at high tides were found in the range of 1 to $1.5 \mathrm{~m}$ and at the lowest tide were exposed to temporal exposure. The distance between seagrass and mangrove forest range was from 250 to $800 \mathrm{~m}$. Seagrass at ST2 at highest tide was found in the depth range of $0.5-1.5 \mathrm{~m}$ and at lowest tide experiencing temporal exposure. The distance between seagrass and coral reefs ranged from 10-50 m. The research was executed from May 2011 to May 2017 with six months interval of observation. $\square$

\section{Seagrass sampling}

Seagrass beds were located on reef flats which were divided into two stations (ST1 and ST2). Each station consisted of 3 quadrant plot sized $0.5 \mathrm{~m} \times 0.5 \mathrm{~m}$. The distance between transects was $50 \mathrm{~m}$, and the distance between quadrant plots was $25 \mathrm{~m}$. Observation of seagrass species covered its species, shoots, frequency, and the length of leaves of each species. The identification of seagrass species was referred to den Hartog (1970), Kuo and McComb (1989), Fortes (1993), Tomascik et al. (1997) and Seagrass Wacth (2010).

\section{Fish sampling}

Fish sampling was carried out by using net set (otoshi ami, trap net, and splint: local name) operated by the local community. Set net is a passive catching device used to block fish migration by using nets that are upheld by bamboo poles to the bottom (Anand et al. 2016) with the end being a trap (Olaniyan 2015). Characteristics of the set net in the study area were pole nets from branches/tree trunks; chamber-crib: $4 \mathrm{~m}^{2}$; chamber-wings: $70 \mathrm{~m}$; main fence: $100 \mathrm{~m}$; and mesh size net: crib: 1 inch; main fence and wing: 1.5 inches. The used set of seagrass bedsmangrove and seagrass beds-coral reef were migrated during high tide. Hauling was done at 24 hours interval. Fish samples were identified by its species and its total length being measured. Identification process was done by referring to Allen (1999); Fishery Research Center (2009); Verhoel (2009); Bergbauer and Kirschner (2011); Jones et al. (2011); Rudi and Mucsin (2011); Wood and Michael (2011); Sharifuddin (2012); Suyatna et al. (2016).

\section{Data analysis}

Data analysis was conducted using descriptive and quantitative approach, i.e., $t$-test $(\alpha=5 \%)$ and correlation $(\alpha=5 \%)$. Seagrass data were analyzed for density (D) (shoot $\mathrm{m}^{-2}$ ) and relative density (RD), frequency (F) and relative frequency (RF), covered (C) and relative covered (RC) (English et al. 1994), important value index (IVI) (Brower et al., 1990). Fish data was analyzed by species composition, Bray Curtis similarity, density (D) (indiv. day $\left.^{-1}\right)$, diversity index $\left(\mathrm{H}^{\prime}\right)$, similarity index (E) and dominance index (C). Correlation between species distribution and habitat was analyzed by Correspondence Analysis (STATISTICA v.8.0)

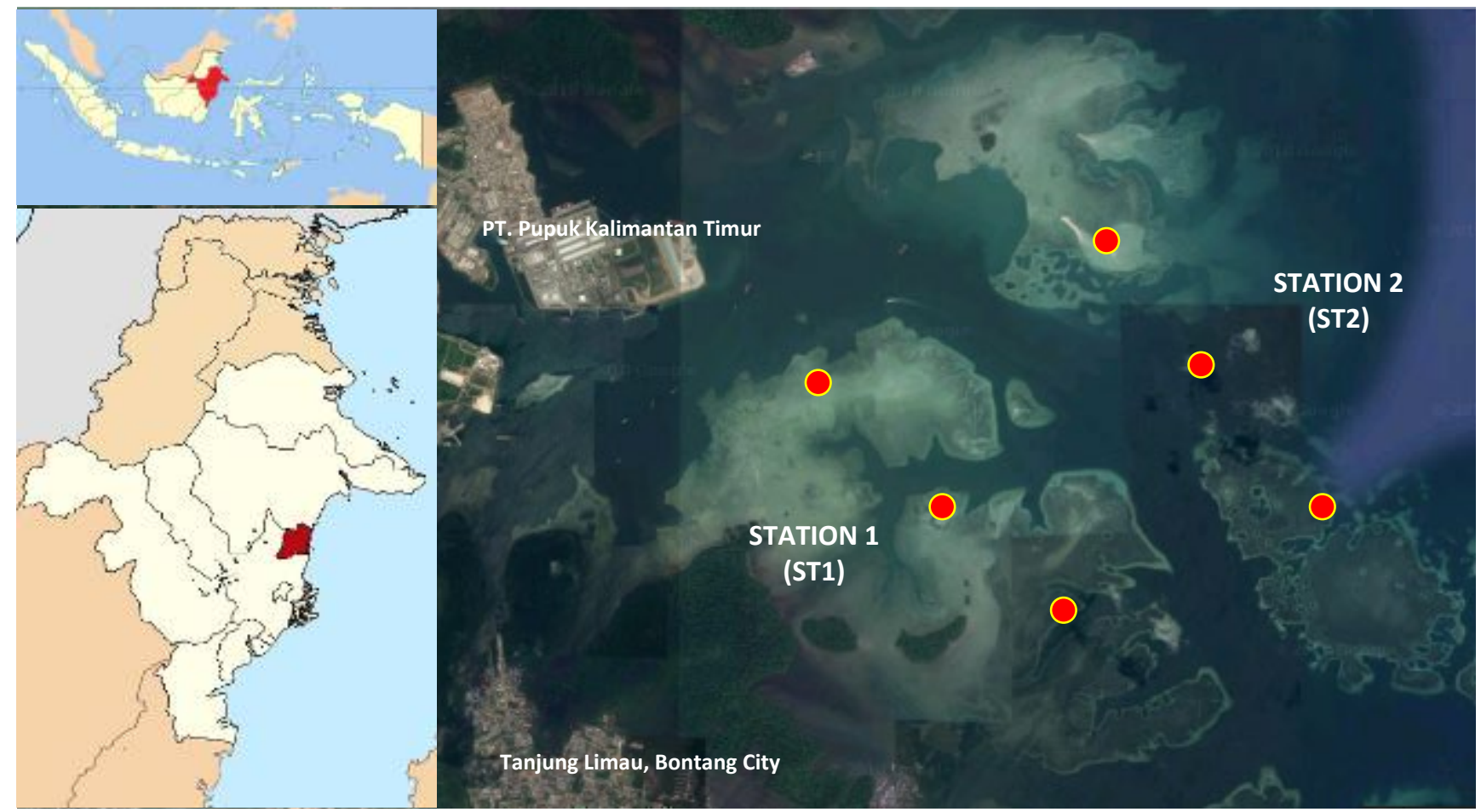

Figure 1. Research location of seagrass beds in coastal water of northern Bontang City, East Kalimantan, Indonesia. ST1: 0009'221'N,

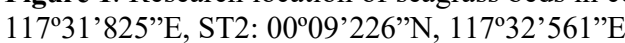




\section{RESULTS AND DISCUSSION}

\section{Distribution of seagrasses}

Seagrass beds in northern Bontang composed of $C$. rotundata, $H$. pinifolia, E. acoroides, $T$. hemprichii, and $H$. minor, while ST 1 composed by $E$. acoroides and $C$. rotundata, and ST 2 composed of the five species. The density of seagrass shoots on ST 1 and ST 2 was similar $\left(\mathrm{P}_{\text {Value }}=0.88\right)$. Important value index shows that $C$. rotundata was more dominant in forming seagrass beds in $\mathrm{ST} 1$ and E. acoroides was dominant in ST2. Both species have a big contribution in forming seagrass beds in northern Bontang. $\square$

Species distribution and fish community structure

The Seagrass bed on both stations provided habitat for

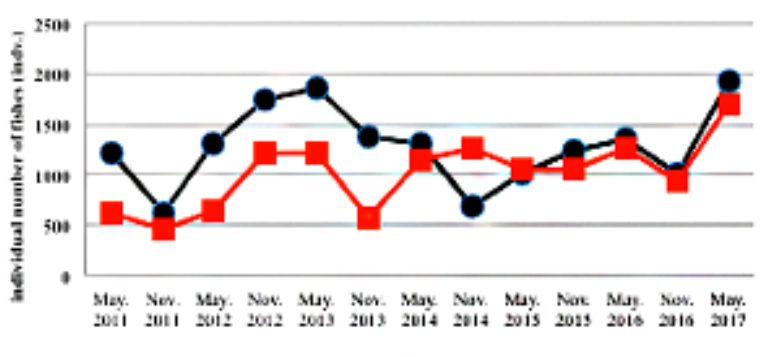

A

ST I

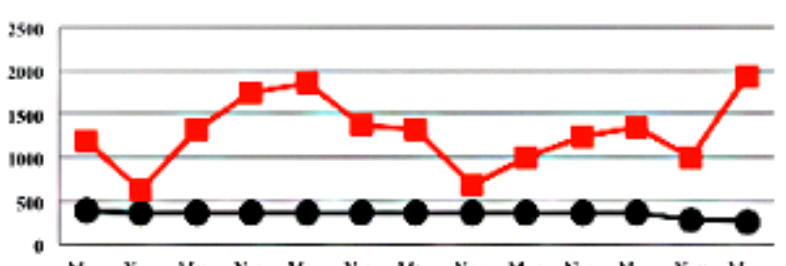

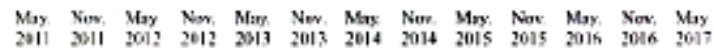

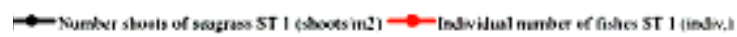

C

ST 1

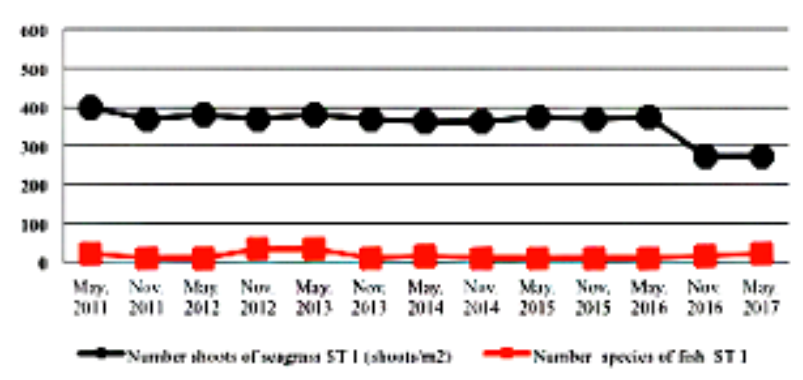

$\mathbf{E}$
112 fish species, with average density in ST 1 was 2087 individuals and 2180 individuals in ST2 (Table 2).

Based on the total number of fish found in ST1 and ST2, we observed the top ten rank of fish species in both stations (Table 2 and 3). The first rank in ST1 with the highest number of population was $G$. flamentous and this species were ranked as the second in ST2. S. doliantus was included in the higher amount of fish species found in both stations, which was ranked as the first and the second in ST2 and ST1, respectively. It was observed that Siganus, Apogon, and Gerres genera were more dominant in forming a fish community in both of the seagrass beds (Table 3). Fish density in both stations was positively correlated with diversity index values (Table 4). The distribution of species tended to be evenly distributed, and no species was dominant (Table 4). $\square$

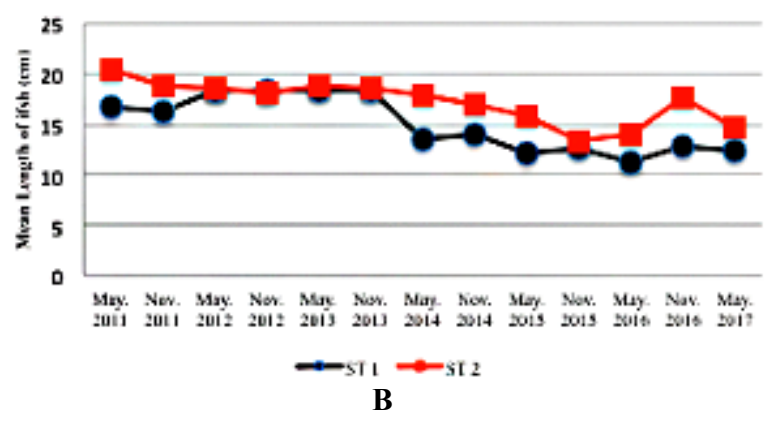

ST 2

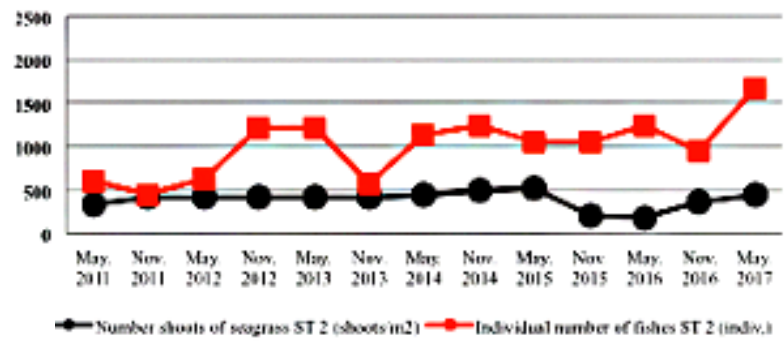

D

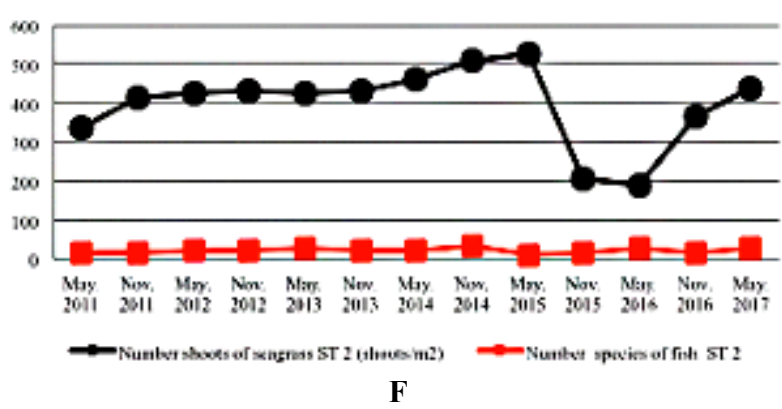

Figure 2. A. Individual number of fish. B. Mean length of fish. C. Number of shoots of seagrass and individual number of fishes at ST1. D. Number of shoots of seagrass and individual number of fishes at ST2. E. Number of shoots of seagrass and number species of fishes at ST1. F. Number shoots of seagrass and number species of fishes at ST2 
Table 1. Length of leaf, density, frequency, covered (mean \pm standard deviation) and important value index of seagrasses in coastal water of northern Bontang City, East Kalimantan, Indonesia

\begin{tabular}{|c|c|c|c|c|c|c|c|c|c|c|c|}
\hline \multirow[t]{2}{*}{ Station } & \multirow[t]{2}{*}{ Species } & \multicolumn{2}{|c|}{$\begin{array}{c}\text { Length of leaf }(\mathrm{cm}) \\
(n=65)\end{array}$} & \multicolumn{2}{|c|}{$\begin{array}{c}\left.\text { Density (shoots } / \mathrm{m}^{2}\right) \\
(n=117)\end{array}$} & \multicolumn{2}{|c|}{$\begin{array}{c}\text { Frequency } \\
(\%)\end{array}$} & \multicolumn{2}{|c|}{$\begin{array}{c}\text { Covered } \\
(\%)\end{array}$} & \multicolumn{2}{|c|}{$\begin{array}{l}\text { Important } \\
\text { value index }\end{array}$} \\
\hline & & Range & Mean & Range & Mean & Range & Mean & Range & Mean & Mean & $\%$ \\
\hline \multirow[t]{2}{*}{ ST1 } & $E . c$ & $43-64.2$ & $54.44 \pm 5.9$ & & & $63-97$ & $85 \pm 0.11$ & $18.42-22.07$ & $20.26 \pm 1.4$ & 1.44 & 47.9 \\
\hline & C. ratundata & $9.5-13.0$ & 11.96 & $197-225$ & 204 & $49-60$ & 53 & $16.12-18.94$ & 17.42 & 1.56 & 52.0 \\
\hline \multirow[t]{5}{*}{ ST2 } & & 19 & 27.6 & & & & & & & 1.36 & 45.17 \\
\hline & C. $r$ & $7.5-1$ & $9.8 \pm$ & $101-179$ & 15 & $32-48$ & & $3.31-6.88$ & 4.28 & 0.86 & 28.50 \\
\hline & T. hemprichii & $6.5-1$ & & & & $6-36$ & & $3-8.28$ & 4. & 0.34 & 11. \\
\hline & H. pinifolia & $4.0-6.0$ & $4.3=$ & $27-$ & & $2-20$ & & -3.12 & & 0.28 & 9. \\
\hline & H. minor & $0.75-0.95$ & $0.77 \pm 0.64$ & $12-49$ & $28 \pm 15.8$ & $2-12$ & $7 \pm 0.04$ & $0.38-1.72$ & $1.09 \pm 0.7$ & 0.16 & 5.3 \\
\hline
\end{tabular}

Table 2. Compositions of species, total length $(\mathrm{cm})$ and density (mean \pm standard deviation) of fishes in coastal water of northern Bontang City, East Kalimantan, Indonesia

\begin{tabular}{|c|c|c|c|c|c|c|c|c|c|c|}
\hline \multirow{3}{*}{ Species } & \multicolumn{3}{|c|}{ ST $1(n=13)$} & \multicolumn{3}{|c|}{ ST $2(n=13)$} & \multicolumn{2}{|c|}{$\begin{array}{c}\text { T-test } \\
\text { (ST1 vs. ST2) }\end{array}$} & \multirow{3}{*}{$\begin{array}{l}\text { Max. TL } \\
\text { (cm) }\end{array}$} & \multirow{3}{*}{$\begin{array}{l}\mathbf{L}_{\mathrm{m}} \\
(\mathbf{c m})\end{array}$} \\
\hline & \multicolumn{2}{|c|}{$\begin{array}{l}\text { Total length } \\
\text { (cm) }\end{array}$} & \multirow{2}{*}{$\begin{array}{c}\text { Density } \\
\left(\text { ind day }^{-1} \text { ) }\right. \\
\text { Mean }\end{array}$} & \multicolumn{2}{|c|}{$\begin{array}{l}\text { Total length } \\
(\mathrm{cm})\end{array}$} & \multirow{2}{*}{$\begin{array}{c}\text { Density } \\
\text { (ind day-1 }{ }^{1} \text { ) } \\
\text { Mean }\end{array}$} & \multirow{2}{*}{ p-values } & \multirow{2}{*}{$\begin{array}{c}\text { Density } \\
\text { p-values }\end{array}$} & & \\
\hline & Range & Mean & & Range & Mean & & & & & \\
\hline Acanthurus albipectoralis & $26.5-28.3$ & $27.3 \pm 0.77$ & $1 \pm 0.55$ & $20-21$ & $20.3 \pm 0.45$ & $1 \pm 1.22$ & 0.000 & 0.335 & $33 * \#$ a & \\
\hline Acreichthys tomentosus $\square$ & 7.5-7.8 & $7.7 \pm 0.13$ & $1 \pm 0.50$ & $5.9-9.1$ & $7.8 \pm 1.03$ & $6 \pm 0.58$ & 0.841 & 0.100 & $12 * \# a$ & \\
\hline Apogon fuscus & $5.7-8.1$ & $7.1 \pm 0.87$ & $7 \pm 0.96$ & - & - & - & - & - & $10 * 11.2^{\# \mathrm{a}}$ & \\
\hline A. kallopterus & $5.9-8.8$ & $7.8 \pm 0.70$ & $51 \pm 1.29$ & $5.1-9.9$ & $7.2 \pm 0.64$ & $18 \pm 3.51$ & 0.437 & 0.126 & $15 * 16^{\# a}$ & \\
\hline A. kiensis & $4.5-10.6$ & $7.7 \pm 0.41$ & $104 \pm 3.37$ & $6.0-11.2$ & $8.3 \pm 0.72$ & $12 \pm 1.15$ & 0.129 & 0.000 & $9 * 8^{\# a}$ & \\
\hline A. poecilopterus & $8.0-9.9$ & $9.3 \pm 0.35$ & $3 \pm 1.15$ & $10.1-11.0$ & $10.4 \pm 0.43$ & $1 \pm 1.1$ & 0.013 & 0.216 & $12 * 14^{\# a}$ & \\
\hline Arothron hispidus & $10.2-28.9$ & $19.6 \pm 6.09$ & $9 \pm 1.01$ & $10.2-25.8$ & $21.1 \pm 4.60$ & $5 \pm 0.82$ & 0.636 & 0.080 & $51 * 50^{\# \mathrm{a}}$ & \\
\hline A. mappa & - & - & - & $21.0-22.0$ & $21.5 \pm 0.41$ & $1 \pm 0.50$ & - & - & $60 * 65^{\# a}$ & \\
\hline A. manillensis & - & - & - & $16.0-16.9$ & $16.4 \pm 0.45$ & $1 \pm 0.58$ & - & - & 31 *\#eg & \\
\hline A. nigropunctatus & $12.8-24.7$ & $21.3 \pm 4.85$ & $2 \pm 0.89$ & $15.7-19.5$ & $18.6 \pm 1.46$ & $1 \pm 0.49$ & 0.178 & 0.109 & $30 * 33^{\# a}$ & \\
\hline A. stellatus & $17.2-40.7$ & $26.3 \pm 3.03$ & $1 \pm 0.58$ & - & - & - & - & - & $90 * 120^{\# \operatorname{deg}}$ & \\
\hline Caesio cuning & $19.3-21.6$ & $20.6 \pm 0.76$ & $3 \pm 0.87$ & $15.2-16.9$ & $15.7 \pm 0.69$ & $2 \pm 0.71$ & 0.000 & 0.053 & $43 * 60^{\# a}$ & \\
\hline C. lunaris & - & - & - & $7.8-8.5$ & $8.2 \pm 0.29$ & $2 \pm 1.26$ & - & - & $38 * 40^{\# a}$ & \\
\hline C. teres & $12.0-13.2$ & $12.7 \pm 0.44$ & $1 \pm 0.55$ & $12.3-13.2$ & $12.9 \pm 0.39$ & $3 \pm 1.41$ & 0.610 & 0.434 & $40^{\# a}$ & \\
\hline Carangoides dinema & - & - & - & $10.0-16.5$ & $14.2 \pm 3.63$ & $8 \pm 1.19$ & - & - & $85^{\# \mathrm{~d}}$ & \\
\hline C. ferdau & $8.7-14.5$ & $11.6 \pm 2.45$ & $1 \pm 0.45$ & - & - & - & - & - & $70^{* \# \mathrm{~d}}$ & \\
\hline C. ignobilis & - & - & - & $12.0-19.0$ & $15.0 \pm 2.94$ & $7 \pm 3.30$ & - & - & $170^{* \# \mathrm{f}}$ & \\
\hline Centriscus scutatus & - & - & - & $10.0-12.5$ & $10.7 \pm 1.24$ & $1 \pm 1.73$ & - & - & $15^{* \# \mathrm{~d}}$ & \\
\hline Cephalopholis sonnerati & $9.0-10.1$ & $9.5 \pm 0.42$ & $1 \pm 0.65$ & $10.8-13$ & $11.9 \pm 0.91$ & $1 \pm 1.41$ & 0.000 & 0.006 & $58 * 57^{\# a}$ & \\
\hline Chanos chanos & $27.3-36.8$ & $32.1 \pm 3.09$ & $1 \pm 0.82$ & - & - & - & - & - & $120 * \mathrm{~d}$ & $98.1^{\#}$ \\
\hline Cheilio inermis & - & - & - & $16.5-29.5$ & $23.0 \pm 4.65$ & $1 \pm 1.22$ & - & - & $50 * \# \mathrm{c}$ & \\
\hline Cheilodipterus quinquelinea & s 9.8-11.2 & $10.8 \pm 0.65$ & $2 \pm 1.19$ & $10.2-11.6$ & $10.9 \pm 0.57$ & $1 \pm 0.82$ & 0.905 & 0.002 & $12 * 13^{\# a}$ & \\
\hline Chelmon rostratus & $8.0-8.7$ & $8.4 \pm 0.24$ & $1 \pm 0.79$ & - & - & - & - & - & $20^{* \# a}$ & \\
\hline Colurodontis paxmani & $5.0-14.5$ & $9.9 \pm 2.27$ & $16 \pm 0.84$ & - & - & - & - & - & $15^{*} 12^{\# \mathrm{c}}$ & \\
\hline Cymbacephalus beauforti & $28.9-34.5$ & $31.7 \pm 1.66$ & $1 \pm 0.90$ & $18.5-25.5$ & $23.5 \pm 2.87$ & $2 \pm 0.84$ & 0.000 & 0.247 & $50^{\# g}$ & \\
\hline Dexillichthys muelleri & - & - & - & $29.0-33.5$ & $30.5 \pm 1.75$ & $1 \pm 0.55$ & - & - & $20 * g$ & \\
\hline Diodon liturosus & $25.1-26.3$ & $25.5 \pm 0.48$ & $1 \pm 0.76$ & $21.0-32.0$ & $25.7 \pm 4.60$ & $2 \pm 0.50$ & 0.911 & 0.247 & $40 * 65^{\# a}$ & \\
\hline Dipterygonatus balteatus & $11.6-12.7$ & $12.1 \pm 0.33$ & $1 \pm 1.07$ & - & - & - & - & - & $14 * \mathrm{a}$ & \\
\hline Epinephelus coioides & $8.5-13.4$ & $10.9 \pm 1.27$ & $3 \pm 0.71$ & $23.5-29$ & $26.3 \pm 3.89$ & $1 \pm 1.41$ & 0.001 & 0.003 & $95 * 120^{\# \text { ae }}$ & $48.3^{\#}$ \\
\hline E. quoуапиs & $15.0-27.0$ & $22.2 \pm 4.67$ & $2 \pm 1.14$ & $10.5-24.5$ & $19.8 \pm 5.46$ & $2 \pm 1.30$ & 0.469 & 0.589 & $35 * 40^{\# \mathrm{f}}$ & \\
\hline E. tukula & - & - & - & $11.0-12.5$ & $11.5 \pm 0.68$ & $1 \pm 1.41$ & - & - & $140 * 200^{\# \mathrm{a}}$ & $99^{\#}$ \\
\hline Escualosa thora & - & - & - & $7.2-11.5$ & $9.2 \pm 1.77$ & $3 \pm 0.82$ & - & - & $10^{\# \mathrm{~d}}$ & \\
\hline Fistularia commersonii & $30.0-50.2$ & $39.8 \pm 11.31$ & $2 \pm 0.96$ & $29.0-32.0$ & $30.5 \pm 1.41$ & $1 \pm 1.41$ & 0.335 & 0.194 & $163^{* \# a d}$ & \\
\hline F. petimba & $62.5-64.8$ & $63.4 \pm 0.70$ & $1 \pm 0.76$ & $48.0-53.7$ & $52.7 \pm 1.16$ & $1 \pm 0.82$ & 0.000 & 0.672 & $185 * 200^{\# \mathrm{a}}$ & \\
\hline Gerres abbreviatus & $8.8-15.5$ & $10.4 \pm 2.26$ & $4 \pm 1.52$ & $10.4-16.5$ & $13.0 \pm 0.84$ & $9 \pm 0.96$ & 0.207 & 0.265 & $30^{\# \mathrm{de}}$ & \\
\hline G. flamentous & $4.2-17.4$ & $12.0 \pm 2.29$ & $370 \pm 4.19$ & $8.8-17.2$ & $13.4 \pm 4.75$ & $101 \pm 4.75$ & 0.061 & 0.000 & $25 * 35^{\# d}$ & $19^{\#}$ \\
\hline Gnathanodon speciosus & $7.1-8.4$ & $7.7 \pm 0.38$ & $1 \pm 0.32$ & $8.6-9,7$ & $9.0 \pm 0.40$ & $1 \pm 0.41$ & 0.000 & 0.337 & $111 * 120^{\# \mathrm{f}}$ & $32.5^{\#}$ \\
\hline Gymnothorax undulatus & - & - & - & $49.4-63.0$ & $56.2 \pm 6.80$ & $1 \pm 1.15$ & - & - & $150 * \# a$ & \\
\hline Halichoeres biocellatus & - & - & - & $8.2-10.0$ & $8.9 \pm 0.65$ & $1 \pm 0.45$ & - & - & $15 * 12^{\# a}$ & \\
\hline Hemirhampus far & - & - & - & $29.0-37.3$ & $32.7 \pm 2.73$ & $6 \pm 0.71$ & - & - & $35 * 45 \#^{\mathrm{d}}$ & range 18 \\
\hline Himantura toshi & 8.7 & 13 & $5 \pm 1.35$ & $38.5-42.5$ & $40.5 \pm 1.63$ & $1 \pm 0.82$ & 0.000 & 0.005 & 180 *beg & \\
\hline us hystrix & $20.7-21.7$ & $21.3 \pm 0.39$ & $1 \pm 0.55$ & $10.8-20.0$ & $14.7 \pm 4.77$ & $3 \pm 1.15$ & 0.017 & 0.864 & $15^{*} 17^{\# \mathrm{~d}}$ & $7.9^{\#}$ \\
\hline Nematalosa come & - & - & - & $13.5-15.0$ & $14.0 \pm 0.67$ & $1 \pm 0.50$ & - & - & $23 * 21^{\# \mathrm{~d}}$ & \\
\hline Lactoria cornuta & - & - & - & $24.6-26$ & $25.3 \pm 0.70$ & $1 \pm 0.71$ & - & - & $46^{* \# \mathrm{cg}}$ & \\
\hline Lagocephalus inermis & $7.5-11.3$ & $8.8 \pm 1.38$ & $4 \pm 0.82$ & - & - & - & - & - & $90^{\# \mathrm{f}}$ & $30^{\#}$ \\
\hline
\end{tabular}




\begin{tabular}{|c|c|c|c|c|c|c|c|c|c|c|}
\hline Leiognathus equulus & $8.8-11.2$ & $9.9 \pm 1.23$ & $2 \pm 0.58$ & $9.2-10.1$ & $9.5 \pm 0.35$ & $1 \pm 0.55$ & 0.5717 & 0.281 & $24 * 28^{\# \text { ae }}$ & $10.7^{\#}$ \\
\hline L. elongatus & $6.0-10.5$ & $8.1 \pm 1.03$ & $3 \pm 1.29$ & $6.0-12.5$ & $9.1 \pm 1.96$ & $37 \pm 0.89$ & 0.4068 & 0.001 & $12^{* \# a}$ & \\
\hline L. fasciatus & $4.9-5.8$ & $5.5 \pm 0.39$ & $2 \pm 0.82$ & $9.7-14.1$ & $12.9 \pm 0.11$ & $5 \pm 1.53$ & 0.000 & 0.353 & $21^{\# \mathrm{de}}$ & \\
\hline Lethrinus lentjan & $6.5-21.5$ & $14.0 \pm 2.05$ & $130 \pm 4.73$ & $8.5-23.0$ & $15.6 \pm 2.30$ & $9 \pm 12.65$ & 0.132 & 0.000 & $50 * 52^{\# \mathrm{ag}}$ & $27.7^{\#}$ \\
\hline L. ornatus & - & - & - & $11.2-18.3$ & $12.7 \pm 1.56$ & $1 \pm 0.71$ & 然 & - & $40 * 45^{\# \mathrm{a}}$ & $20^{\#}$ \\
\hline L. variegatus & $6.8-9.0$ & $9.2 \pm 1.96$ & $3 \pm 1.26$ & $7.6-18.2$ & $12.9 \pm 1.88$ & $29 \pm 1.05$ & 0.016 & 0.006 & 20 *\#acg & \\
\hline Liza vaigiensis & $43.1-47.0$ & $45.0 \pm 1.95$ & $1 \pm 0.58$ & - & - & - & - & - & $55 * 63^{\# \text { ade }}$ & \\
\hline Lutianus lutjanus & $14.1-14.9$ & $14.3 \pm 0.31$ & $1 \pm 0.82$ & $11.8-12.0$ & $11.9 \pm 0.10$ & $1 \pm 1.15$ & 0.485 & 1.000 & $30 * \# a$ & \\
\hline L. bitaeniatus & $9.8-13.4$ & $11.6 \pm 1.30$ & $9 \pm 2.17$ & $14.2-15.5$ & $14.8 \pm 0.56$ & $1 \pm 0.82$ & 0.002 & 0.010 & 30 *\#af & \\
\hline L. decussatus & $10.7-13.2$ & $12.0 \pm 1.13$ & $5 \pm 1.29$ & $11.1-13.7$ & $12.6 \pm 1.10$ & $4 \pm 1.41$ & 0.009 & 0.731 & $30 * \#$ af & \\
\hline L. fulviflamma & $10.6-17.2$ & $13.8 \pm 3.31$ & $1 \pm 1.15$ & $20.1-21$ & $20.6 \pm 0.46$ & $1 \pm 0.96$ & 0.533 & 0.550 & $35^{* \# a}$ & $17.1^{\#}$ \\
\hline L. fulvus & $13.5-21.0$ & $15.5 \pm 2.22$ & $65 \pm 3.08$ & $10.6-19.8$ & $16.2 \pm 1.39$ & $5 \pm 0.89$ & 0.000 & 0.000 & $40 * \# a$ & $22.5^{\#}$ \\
\hline L. johnii & $12.4-15.5$ & $14.3 \pm 1.48$ & $2 \pm 5.52$ & te 1 & - & - & - & - & $70 * 97^{\# \mathrm{be}}$ & $39^{\#}$ \\
\hline L. lunulatus & - & - & - & $22.0-24.2$ & $23.1 \pm 0.90$ & $1 \pm 0.82$ & - & - & $35 * 40^{\# \mathrm{a}}$ & \\
\hline L. quinquelinetus & $10.7-12.3$ & $11.1 \pm 0.67$ & $1 \pm 0.55$ & $16.5-17.7$ & $17.0 \pm 0.43$ & $1 \pm 0.55$ & 0.000 & 1.000 & $38^{* \# a}$ & \\
\hline Megalops cyprinoides & $30.1-31.0$ & $30.5 \pm 0.35$ & $1 \pm 0.52$ & $25.2-29.5$ & $27.5 \pm 1.54$ & $1 \pm 0.45$ & 0.001 & 0.318 & 150 *\#be & $25^{\#}$ \\
\hline Monodactvlus argenteus & $15.3-16.7$ & $16.0 \pm 0.41$ & $1 \pm 0.53$ & - & - & - & - & - & $27 * \#$ be & $13^{\#}$ \\
\hline Mulloidichthys vanicolensis & - & - & - & $17.1-19.3$ & $18.0 \pm 0.80$ & $1 \pm 0.71$ & - & - & $33 * 38^{\# \mathrm{ag}}$ & $24^{\#}$ \\
\hline Mugil cephalus & $17.5-18.3$ & $18.0 \pm 0.24$ & $1 \pm 0.79$ & - & - & - & - & - & $100^{\# \mathrm{~d}}$ & $35.4^{\#}$ \\
\hline Pomacentrus milleri & - & - & - & $9.8-11.6$ & $10.7 \pm 0.65$ & $1 \pm 1.22$ & - & - & $13 * a$ & \\
\hline Paramonacanthus choirochephalu. & $9.0-19.5$ & $11.8 \pm 2.19$ & $6 \pm 1.73$ & $13.4-18.0$ & $16.0 \pm 1.78$ & $3 \pm 1.50$ & 0.037 & 0.320 & $14 * \mathrm{~g}$ & \\
\hline P. filicauda & - & - & - & $7.0-13.5$ & $10.0 \pm 2.57$ & $2 \pm 0.58$ & - & - & $22^{* \# g}$ & \\
\hline Parapercis tetracanata & $11.7-12.9$ & $12.4 \pm 0.50$ & $1 \pm 0.82$ & $6.0-13.4$ & $7.9 \pm 3.20$ & $2 \pm 1.34$ & 0.029 & 0.152 & $29^{\# \mathrm{~d}}$ & \\
\hline Paraplotosus albilabris & - & - & - & $8.7-16.5$ & $=1.93$ & $63 \pm 1.40$ & - & - & $134 * \# a$ & \\
\hline Pardachirus pavoninus & $13.0-20.4$ & $17.4 \pm 3.84$ & $2 \pm 3.79$ & $12.0-17.3$ & $14.7 \pm 1.88$ & $1 \pm 1.41$ & 0.219 & 0.383 & $25^{* \# g}$ & \\
\hline Parupeneus margaritatus & 13.7 & $12.2 \pm 1.11$ & $1 \pm 2.45$ & $10.6-22.7$ & $17.3 \pm 5.40$ & $1 \pm 0.71$ & 0.117 & 0.723 & $23^{\# \mathrm{a}}$ & \\
\hline P. indicus & - & - & - & $12.5-15.0$ & $13.8 \pm 0.80$ & $1 \pm 1.03$ & - & - & $40 * 45^{\text {\#ag }}$ & \\
\hline Pelates quadrilineatus & - & - & - & $9.0-13.0$ & $11.1 \pm 1.62$ & $10 \pm 1.72$ & - & - & $20 * 30^{\# \mathrm{de}}$ & \\
\hline Pentapodus caninus & $11.5-16.0$ & $13.1 \pm 1.47$ & $3 \pm 2.52$ & $11.0-14.8$ & $13.0 \pm 1.07$ & $9 \pm 1.64$ & 0.221 & 0.556 & $20 * 35^{\# \mathrm{a}}$ & \\
\hline P. bifasciatus & $6.5-16.0$ & $13.0 \pm 1.93$ & $21 \pm 5.50$ & 6.5 & $14.1 \pm 1.10$ & $25 \pm 1.72$ & 0.900 & 0.013 & $18 * 20^{\# \mathrm{a}}$ & \\
\hline Pentaprion longimanus & - & - & - & $16.3-18.1$ & $14.8 \pm 1.43$ & $1 \pm 0.71$ & - & - & $15^{* \# \mathrm{dg}}$ & \\
\hline Platax sp. & $9.0-17.0$ & $14.7 \pm 3.35$ & $5 \pm 2.06$ & $18.0-20.5$ & $17.2 \pm 4.61$ & $6 \pm 1.33$ & 0.382 & 0.566 & $50 * \mathrm{a}$ & \\
\hline Platybelone arga & - & - & - & 42.3 & $39.7 \pm 1.36$ & $1 \pm 0.82$ & - & - & $40 * 45^{\# \mathrm{f}}$ & \\
\hline Pseudomonacan & & - & - & 17.1 & 0.54 & 64 & - & - & $24 * c$ & \\
\hline Rastrelliger bra & 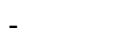 & _ & _ & & 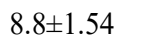 & $1 \pm 0.58$ & - & - & $34.5^{\# \mathrm{eh}}$ & $17^{\#}$ \\
\hline R. kanagurata & $17.7-21.3$ & $19.6 \pm 1.80$ & $1 \pm 1.53$ & 30.0 & $23.8 \pm 6.25$ & $1 \pm 1.41$ & 0.326 & 0.147 & $35^{* \wedge a d}$ & $23^{\wedge}$ \\
\hline R. faughni & $19.1-20.1$ & $19.7 \pm 0.39$ & $1 \pm 0.82$ & -1 & - & - & - & - & $20^{\text {\#h }}$ & \\
\hline Sardinella gibbosa & - & - & - & 6. & 8.9 & $13 \pm 1.29$ & - & - & $19 * 17^{\# \mathrm{~d}}$ & $12.8^{\#}$ \\
\hline Scarus chameleon & $13.1-13.8$ & $13.5 \pm 0.29$ & $1 \pm 0.50$ & 31.6 & 6.01 & $1 \pm 1.15$ & 0.134 & 0.438 & $28 * a$ & \\
\hline S. dimidiatus & - & - & - & & 0.10 & $1 \pm 0.71$ & & & $34 * 40^{\# a}$ & \\
\hline ceps & 9.5 & 0.17 & $1 \pm 0.45$ & 9.4 & .94 & 63 & 0.050 & 0.040 & $30 * 45^{\# \mathrm{a}}$ & \\
\hline bban & - & - & - & 49.0 & .15 & $1 \pm$ & - & - & $100 * \mathrm{ac}$ & ge 4 \\
\hline Scolopsis auratu & - & _ & _ & 21.0 & \pm 0.75 & $1 \pm 1.53$ & - & - & $22 * 24 \# a$ & \\
\hline S. ciliatus & $9.0-14.3$ & $13.8 \pm 1.01$ & $11 \pm 8.02$ & $10.1-21.0$ & $14.8 \pm 2.46$ & $50 \pm 1.13$ & 0.834 & 0.000 & $16 * 25^{\# \mathrm{~g}}$ & \\
\hline S. margaritifer & $8.5-18.1$ & $13.1 \pm 0.60$ & $7 \pm 1.15$ & $11.6-20.0$ & $15.2 \pm 2.52$ & $5 \pm 1.26$ & 0.226 & 0.531 & $20 * 28^{\# \mathrm{f}}$ & \\
\hline S. taeniopterus & $14.2-15.2$ & $14.6 \pm 0.36$ & $1 \pm 0.89$ & $12.0-20.0$ & $16.0 \pm 3.61$ & $2 \pm 0.58$ & 0.385 & 0.256 & $23 * 30^{\# f}$ & \\
\hline Scomberoides tala & $13.8-14.4$ & $14.1 \pm 0.22$ & $1 \pm 0.55$ & -31.9 & \pm 0.54 & $1 \pm 0.50$ & 0.000 & 0.603 & $75^{*} 70^{\# \mathrm{c}}$ & \\
\hline Selar boops & $11.0-15.7$ & $14.8 \pm 2.10$ & $7 \pm 0.58$ & $11.0-17.5$ & $13.7 \pm 2.29$ & $32 \pm 1.33$ & 0.415 & 0.003 & $25^{* \# \mathrm{c}}$ & \\
\hline S. crumenthalmops & - & - & - & $8.0-18.0$ & $13.7 \pm 5.13$ & $1 \pm 0.58$ & - & - & $30 * 70^{\# \mathrm{c}}$ & $17^{\#}$ \\
\hline hagus argus & 2.9 & 30 & $1+055$ & - & $10.1+5.10$ & - & - & - & $33 * 38^{\# \text { be }}$ & rang \\
\hline atus $\square$ & .5 & 30 & 2.80 & $11.1-29.0$ & $16.6 \pm 3.84$ & $77 \pm 1.38$ & 0.886 & 0.049 & $p^{\# \mathrm{cg}}$ & $11.6^{\#}$ \\
\hline linus & 8.1 & $8.2 \pm 0.08$ & $1 \pm 0.82$ & - & - & - & - & - & $28 * 35^{\# \mathrm{ac}}$ & \\
\hline S. doliantus & $6.5-21.0$ & $14.4 \pm 3.63$ & $187 \pm 2.96$ & & \pm 1.41 & $297 \pm 7.14$ & 0.263 & 0.182 & $30 * 25^{\mathrm{Ha}}$ & \\
\hline S. fuscescens & - & - & - & $11.7-14.5$ & $13.6 \pm 1.62$ & $3 \pm 1.15$ & - & - & $41 * \mathrm{c}$ & \\
\hline S. guttatus & $14.5-25.5$ & $19.6 \pm 3.40$ & $29 \pm 1.75$ & $12.5-25.0$ & $19.1 \pm 2.87$ & $72 \pm 5.61$ & 0.777 & 0.000 & $53^{* \# a d}$ & \\
\hline S. vermiculatus & - & - & - & $24.6-27.3$ & $26.2 \pm 1.31$ & $1 \pm 0.96$ & - & - & $45^{* \# a}$ & \\
\hline S. virgatus & - & - & - & $9.7-12.3$ & $11.2 \pm 0.26$ & $4 \pm 1.41$ & - & - & 30 *a & \\
\hline Sphyraena jello & - & - & - & $27.5-70.0$ & $46.9 \pm 1.13$ & $1 \pm 0.82$ & - & - & $150 * \#$ def & \\
\hline S. qenie & $15.5-25.5$ & $23.5 \pm 0.47$ & $4 \pm 1.71$ & $18.5-30.0$ & $24.0 \pm 4.61$ & $2 \pm 0.96$ & 0.284 & 0.238 & $90 * 170^{\# \mathrm{~d}}$ & \\
\hline Sphaeramia orb & - & - & - & & $8.7 \pm 2.37$ & $5 \pm 4.36$ & - & - & $11.5 * 10^{\# \mathrm{bd}}$ & \\
\hline Stolephorus indicus & & 9.2 & 10 & $6.5-13.0$ & $9.9 \pm 2.07$ & $15 \pm 1.11$ & 0.517 & 0.444 & $16^{*} 15.5^{\# \mathrm{de}}$ & $9^{\#}$ \\
\hline Synanceia verrucosa & 15.8 & $15.0 \pm 0.64$ & $1 \pm 0.45$ & - & - & - & - & - & $35 * 40^{\# a}$ & range $24^{\#}$ \\
\hline Taeniura lymma & - & - & - & $43.0-51.0$ & $47.0 \pm 3.74$ & $1 \pm 1.41$ & - & - & $70 * a g$ & $20.3^{\#}$ \\
\hline Thryssa hamiltonii & $20.0-20.9$ & $20.5 \pm 0.34$ & $1 \pm 0.89$ & - & - & - & - & - & $25 * 27^{\# \text { beg }}$ & \\
\hline Tylosurus gavialoi & -1 & - & - & $30.5-33.7$ & $32.1 \pm 1.60$ & $1 \pm 0.58$ & - & - & $130 * \mathrm{~d}$ & \\
\hline Upeneus tragula & 18.2 & $14.7 \pm 0.23$ & $4 \pm 2.63$ & $13.0-18.1$ & $16.3 \pm 1.90$ & $5 \pm 1.81$ & 0.262 & 0.613 & $30 * 25^{\# \mathrm{cg}}$ & \\
\hline U. vittatus & $11.5-13.4$ & $13.0 \pm 0.98$ & $7 \pm 1.00$ & $9.0-15.0$ & $11.0 \pm 2.83$ & $4 \pm 2.52$ & 0.170 & 0.398 & $28^{* \# \mathrm{cg} g}$ & \\
\hline Mean & & & 1280 & & & 1013 & & & & \\
\hline
\end{tabular}

Note: *: Allen (1999). Max.TL: Maximum total length. Habitat: a. Coral reefs, b. Mangrove, c. Seagrass beds-weedy area, d. Coastal water. \# : www.fishbase.org $\mathrm{L}_{\mathrm{m}}$ : Length maturity, e. Estuaries, f. Inshore/offshore reefs, g. Mud/sand bottom. ^: FAO 2013 (www.fao.org/fishery), h. pelagic-neritic 
Table 3. Top ten ranks of fish species based on the amount of fish found in ST1 and ST2 of the coastal water of northern Bontang City, East Kalimantan, Indonesia

\begin{tabular}{|c|c|c|c|c|c|c|c|}
\hline \multicolumn{4}{|c|}{ ST1 } & \multicolumn{4}{|c|}{ ST2 } \\
\hline \multirow[b]{2}{*}{ Rank } & \multirow[b]{2}{*}{ Species $\square$} & \multicolumn{2}{|c|}{ Percentage (\%) } & \multirow[b]{2}{*}{ Rank } & \multirow[b]{2}{*}{ Species $\square$} & \multicolumn{2}{|c|}{ Percentage (\%) } \\
\hline & & $\begin{array}{l}\text { Mean } \\
\text { Number } \\
\text { of ind. }\end{array}$ & Contributions & & & $\begin{array}{c}\text { Mean } \\
\text { number of } \\
\text { ind. }\end{array}$ & Contribution \\
\hline 1 & Gerres flamentous & 32.29 & 19.25 & 1 & Siganus doliantus & 36.51 & 18.76 \\
\hline 2 & Siganus doliantus & 20.85 & 12.43 & 2 & Gerres flamentous & 13.87 & 7.13 \\
\hline 3 & Apogon kiensis & 15.45 & 9.21 & 3 & Siganus guttatus & 13.74 & 7.06 \\
\hline 4 & Lethrinus lentjan & 15.07 & 8.98 & 4 & Siganus canaliculatus $\square$ & 12.22 & 6.28 \\
\hline 5 & Siganus canaliculatus $\square$ & 14.03 & 8.37 & 5 & Paraplotosus albilabris & 9.93 & 5.10 \\
\hline 6 & Apogon kallopterus & 12.38 & 7.38 & 6 & Scolopsis ciliatus & 9.10 & 4.68 \\
\hline 7 & Lutjanus fulvus & 6.88 & 4.10 & 7 & Pelates quadrilineatus & 7.06 & 3.63 \\
\hline 8 & Siganus guttatus & 5.27 & 3.14 & 8 & Leiognathus elongatus & 6.76 & 3.47 \\
\hline 9 & Pentapodus bifasciatus & 4.10 & 2.44 & 9 & Selar boops & 5.68 & 2.92 \\
\hline 10 & Stolephorus indicus & 2.89 & 1.72 & 10 & Apogon kallopterus & 5.65 & 2.90 \\
\hline
\end{tabular}

Table 4. Density, diversity, similarity, dominance (mean \pm standard deviation) and correlation of fish found in ST1 and ST2 of the coastal water of northern Bontang City, East Kalimantan, Indonesia

\begin{tabular}{|c|c|c|c|c|c|c|c|c|c|c|c|c|}
\hline \multirow{3}{*}{ Station } & \multirow{2}{*}{\multicolumn{3}{|c|}{ Density (indv. day ${ }^{-1}$ ) }} & \multicolumn{9}{|c|}{ Index } \\
\hline & & & & & Diversity & & & Similarity & & & Dominance & \\
\hline & Range & Mean & $\mathbf{r}$ & Range & Mean & $\mathbf{r}$ & Range & Mean & $\mathbf{r}$ & Range & Mean & $\mathbf{r}$ \\
\hline ST1 & $623-1928$ & $1280 \pm 401.2$ & $+) 0.47$ & $0.58-2.84$ & $1.74 \pm 0.66$ & $(+) 0.61$ & $0.41-0.81$ & $0.62 \pm 0.18$ & $+) 0.11$ & $0.10-0.79$ & $0.33 \pm 0.20$ & $(+) 0.42$ \\
\hline ST2 & $457-1686$ & $1013 \pm 358.4$ & & $1.39-2.98$ & $2.36 \pm 0.43$ & & $0.49-0.87$ & $0.76 \pm 0.11$ & & $0.07-0.42$ & $0.16 \pm 0.09$ & \\
\hline
\end{tabular}

Correspondence analysis showed three groups of species-associated, i.e., (i) 29 species were closely associated with ST1 (A. kallopterus, A. poecilopterus, A. nigropunctatus, $C$. lunaris Cymbacephalus beauforti, $F$. petimba, G. undulatus, H. hystrix, N. come, L. equulus, $L$. elongatus, L. lentjan, L. bitaeniatus, L. quinquelinetus, $M$. cyprinoides, $M$. argenteus, $P$. caninus, $R$. kanagurata, $R$. faughni, S. ciliatus, S. margaritifer, S. margaritifer, S. doliantus, S. guttatus, T. gavialoi, U. tragula, U. vittatus), (ii) 32 species were closely associated with ST2 ( $A$. stellatus, $C$. teres, $C$. dinema, $C$. inermis, $E$. coioides, $E$. tukula, E. thoracata, G. speciosus, H. biocellatus, H. far, $H$. toshi, $L$. fasciatus, $L$. fulviflamma, $L$. fulvus, $L$. lunulatus, $M$. vanicolensis, P. milleri, P. filicauda, $P$. albilabris, $P$. margaritatus, $P$. quadrilineatus, Platax sp., P. platyura, P. macrrurus, S. chameleon, S. globiceps, $S$. gobban, S. auratus, Selar boops, S. vermiculatus, $S$. indicus, T. lymma), (iii) and 51 species were spread in both stations (Figure 3.A and 3.B).

\section{Discussion}

\section{Seagrass beds}

Seagrass beds ST1 were constituted by two species which was dominated by the density of $C$. rotundata and ST2 was constituted by five species dominated by $E$. acoroides (Table 1). Based on the morphometric analysis, $E$. acoroides had the biggest size than the rest of the species (Table 1). Thus, its existence could protect other smaller seagrass species against stream and waves
(Fonseca and Cahalan 1992; John et al. 2015). Seagrass beds provide habitat for a fish community (Phinrub et al. 2014), the existence of seagrass stand especially $E$. acoroides (Lotuconsina et al. 2012; Ambo-Rappe et al., 2013) and $C$. rotundata is a vegetative option chosen by the fish species to associate. The association can be seen especially in ST2 where the density of the seagrass was experiencing a decline, especially $H$. pinifolia, $T$. hemprichii, and $H$. minor but the number of fish species available in ST2 was consistent, and so was the fish species in ST1 which was likely stable (Figure 2.E and 2.F).

\section{Distribution and fish community structure}

Spatial patterns. Seagrass beds ST1 that was formed by two seagrass species contributed in providing habitat for 73 fish species or $51.09 \%$ of total amount of fish species (Table 2). The seagrass beds ST2 formed by five species of seagrass contributed in providing habitat for 95 fish species or $48.91 \%$ of total amount of caught fishes (Table 2). Specifically, in ST1, only 17 species were found. These species share a relatively similar total length (Table 2 ). The total length of a species was relatively shorter than that appeared in South-East Asia (Allen 1999) and tended to be in the juvenile phase or immature, such as A. fuscus, $L$. vaigiensis and T. hamltonii, A. stellatus, C. ferdau, Chanos chanos, C. rostratus, C. paxmani, Lagocephalus sp., L. johnii, $M$. argenteus, $M$. cephalus, $R$. tayenus, $S$. argus, $S$. corallinus, and $S$. verrucosa (Table 2). There were 39 fish species found in ST2 which had relatively similar total 
length. Some of the fish species tended to be in the juvenile phase or immature, such as A. mappa, A. manillensis, $C$. dinema, C. ignobilis, E. tukula, L. cornuta, L. ornatus, $P$. albilabris, P. indicus, P. quadrilineatus, S. gibbosa, S. dimidiatus, S. gobban, $S$. auratus, $S$. crumenthalmops, $S$. virgatus, S. jello, T. Gavialoi (Table 2). Meanwhile, the same species found in both stations showed that (Table 2), there was a tendency of differences in the total length and a significant difference in the number of species. The same species found in both stations were insignificantly different in their total length but significantly differed in their density (A. hispidus, Caesio sp., C quinquelineatus, L. equulus, L. fulviflamma, $P$. bifasciatus, S. ciliatus, $S$. canaliculatus, $S$. indicus). It also noted that in ST1 the species density was higher and total length shorter than that in ST2 (Figure 3.B). This included the total length of fishes ranked in top 10 in which the total length of $G$. filamentosus in ST1 was shorter than in ST2 (Table 2 and 3 ). This shows that the characteristic of the land compiler seagrass species in both stations serves not only as a space for biology phase from each species but also as a biomass distribution (Figure 3.A and 3.B) (ter Braak 1987; Ludwig and Reynolds 1988; ter Braak and Verdonschot 1995).

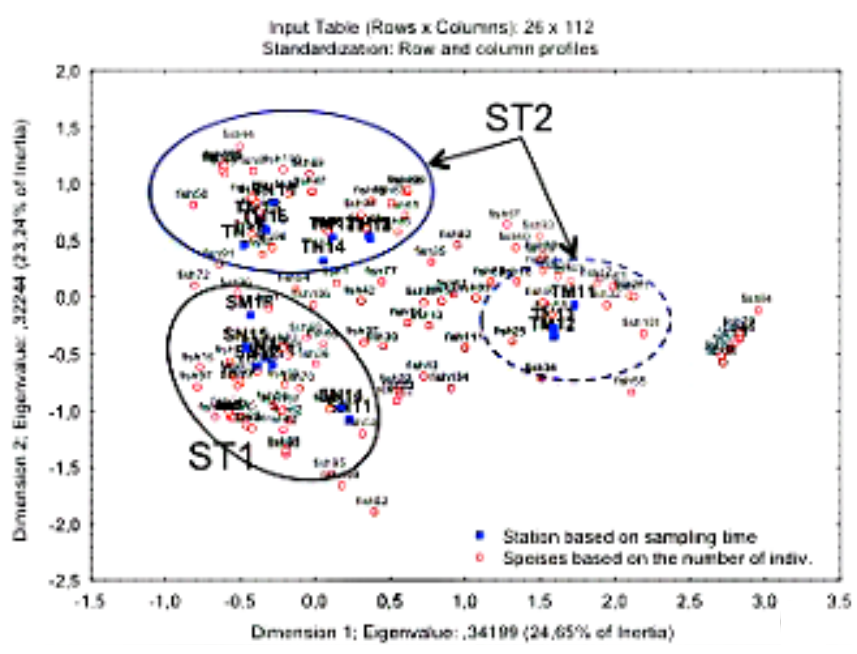

$\mathbf{A}$

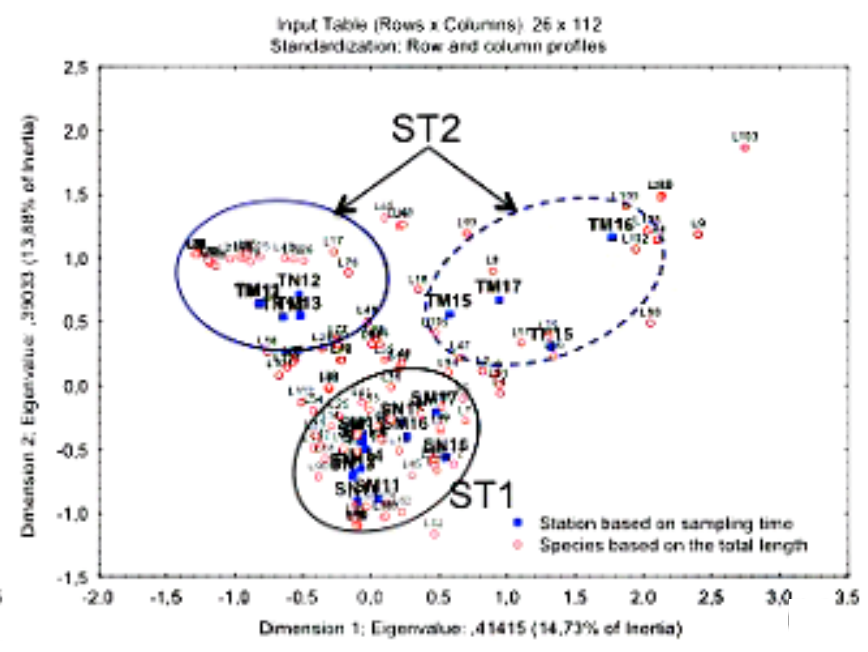

B

Figure 3. Correspondence analysis with $90 \%$ of inertia: A. Distributions of species based on the number of individual vs. station based on sampling time; B. distributions of species based on the total length vs. station based on sampling time. Fish1-fish112 represents number of individual of species and L1-L112 represents total length of species

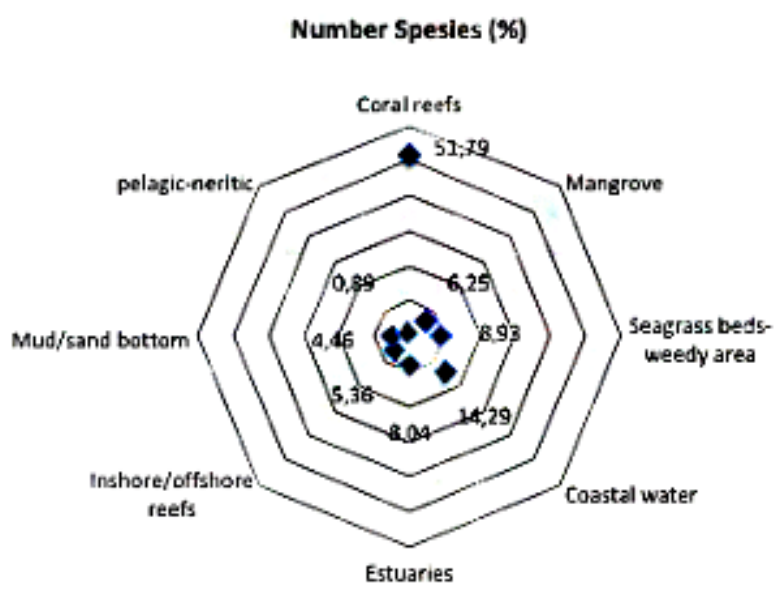

A

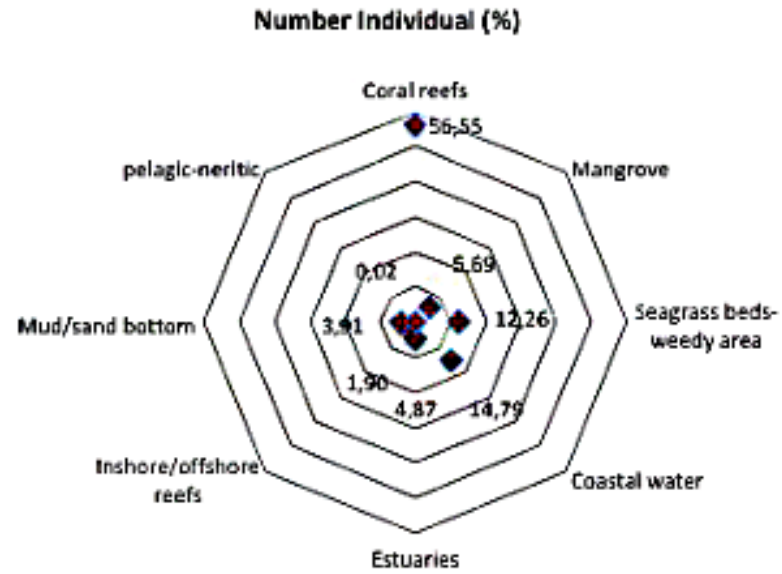

B

Figure 4. The estimated contribution for a number of species (A) and a number of fish (B) on the mature phase from seagrass beds to coastal water of northern Bontang City, East Kalimantan, Indonesia 
Table 3 shows that the contributions of $S$. doliantus $(31.19 \%)$, G. filamentosus $(26.38 \%), S$. canaliculatus (14.65\%), A. kallopterus (10.28\%), S. guttatus (10.20\%) were larger than other species stated on both stations in forming a fish community of seagrass beds. A. kiensis, $L$. lentjan, L. fulvus, $P$. bifasciatus and $S$. indicus tended to be critical species on ST1 while $P$. albilabris, $S$. ciliatus, $P$. quadrilineatus, L. elongatus, and $S$. boops tended to be key species on ST2.

The density of fishes tended to be higher in ST1 compared to ST2, showing that the abundance relationship between the two stations is positively correlated (Table 4). This fact indicated that when the population of fish species in ST1 is higher, the number of fish species population in ST2 will also increase and vice versa (Figure 2.A). Study based on the total length size of the fishes showed that the average total length of fishes in ST1 tended to be shorter than that in ST2 (Table 2). It shows that fishes with smaller sizes tended to be found in ST1 and fishes with larger sizes from the same species tended to be found in ST2 (Figure 2.B). The selection of habitat characteristics by the fish communities in the seagrass is related to seagrass beds (McCloskey et al. 2015) as nursery ground especially in the juvenile phase (Jones 2014) and dietary changes (Lepoint et al. 2016) in the adult (Dromard et al. 2017) or migratory phase with regard to age (phase) (Ambo-Rappe et al. 2013; Honda et al. 2013; Phinrub et al. 2014; Oliveira et al. 2016).

Temporal patterns. Species of predator fishes found in ST1 (Genera: Acreichthys, Arothron, Carangoides, Cephalopholis, Dexillichthys, Diodon, Epinephelus, Gymnothorax, Hemirhampus, Himantura, Leiognathus, Lethrinus, Lutjanus, Mulloidichthys, Paramonacanthus, Paraplotosus, Parupeneus, Pelates, Pentapodus, Pentaprion, Platax, Platybelone, Pseudomonacanthus, Rastrellinger, Scarus, Scolopsis, Scomberoides, Taeniura, and Upeneus) were longer in size than that found in the ST2. This condition shows that the existence of seagrass beds in ST1 was more likely to become the place for smaller fishes to find shelter and food (Cucio et al. 2016); their existence attracts predator fishes originated from coral reef ecosystem (Verweij et al. 2008). This shows the tendency of the ST1 role as a nursery ground and ST2 as a feeding ground. This fact confirmed the role of seagrass beds as a nursery grounds (Verweij et al. 2008; Campbell et al. 2011) and a feeding ground (Hantanirina and Benbow 2013; Blandon and zu Ermgassen 2014) and on that mature phase in part of the big fish species will be going to other ecosystem which is specific to a coral reef as their habitat (Allen 1999).

The above phenomenon shows a distribution or migration of the same fish species which made ST1 and ST2 as their habitat at different times. This condition can be seen from diversity index value, similarity, and dominance values which showed a positive correlation between two stations (Table 4). The high level of diversity index value and similarity as well as the lower level of dominance index value in both stations shows that seagrass beds had played an important role in preserving fish biodiversity in coastal water (Nadiarti et al. 2012) which was confirmed from the fish connectivity (Unsworth et al. 2007; Waycott et al. 2009) in both stations.

The estimated contribution for a number of species and a number of fish on the mature phase from seagrass beds to coastal water, shows that seagrass bed contributed significantly not only to the coral reefs but also to coastal water, mangrove, estuaries, inshore/offshore reefs, $\mathrm{mud} / \mathrm{sand}$ bottom, and pelagic-neritic), while species staying permanently in seagrass beds was over $8.9 \%$ (Figure 4). Physical interaction among fishes in the two stations had made seagrass beds as a buffer zone for physical distribution of fish species found in the coastal ecosystem. The fact shows the existence of seagrass beds as an ecosystem can connect fish species between mangrove and coral reef ecosystem as well as in other ecosystems. This condition gives us the ability to forecast the impact of losing seagrass beds to fish species biodiversity in the coastal ecosystem. $\square$

\section{ACKNOWLEDGEMENTS}

Authors acknowledged the facility supports from PPLH of Universitas Mulawarman, Samarinda, Indonesia. We gratefully thank Adi Susanto, Lily Inderia Sari, Arini, Ade Meiliyana, Yuliani, Syaripudin, Amir Hamzah, Akhmad, and Budi for their help during fieldwork and fish identification. $\square$

\section{REFERENCES}

Allen G. 1999. Marine Fisher of South-East Asia. Periplus Edition, Singapore.

Ambo-Rappe A, Nessa MN, Latuconsina H, Lajus DL. 2013. Relationship between the tropical seagrass bed characteristics and the structure of the associated fish community. Open J Ecol 3: 331-342.

Anand M, Divya G, Beevi H. 2016. Lead-trap Net Fishing in the Gulf of Mannar region, Southeast Coast of India. Indian J Geo-Marine Sci 45 (7): 863-868.

Bahlmann E, Weinberg I, Lavri`c JV, Eckhardt T, Michaelis W, Santos R, Seifert R. 2015. Tidal controls on trace gas dynamics in a seagrass meadow of the Ria Formosa lagoon (southern Portugal). Biogeosciences 12: 1683-1696.

Berbauer M, Kirschner M. 2011. Tropical Marine Life of the Indo-Pacific Region. John Beaufoy Publishing, UK.

Billah MM, Zamal H, Kamal AHM, Hoquef ATMR, Rahman MM, Hoque MM, Akhtar A, Hoque MN. 2016. Saltmarsh and seagrass beds on the south-eastern coast of Bangladesh: vegetation characteristics and adjacent fisheries diversity. Zool Ecol 4: 1-10.

Blandon A, zu Ermgassen PSE. 2014. Quantitative Estimate of Commercial Fish Enhancement by Seagrass Habitat in Southern Australia. Estuar Coast Shelf Sci 141: 1-8

Brower JE, Zar JH, Von Ende C. 1990. General Ecology. Field and Laboratory Methods. Wm. C. Brown Co. Publ., Dubuque, Iowa.

Campbell SJ, Kartawijaya T, Sabarini EK. 2011. Connectivity in reef fish assemblages between seagrass and coral reef habitats. Aquat Biol 13: 65-77.

Carrol JM, Peterson BJ. 2013. Ecological trade-offs in seascape ecology: bay scallop survival and growth across a seagrass seascape. Landsc Ecol 28: 1401-1413.

Coles R.G, Long WJL, Watson RA, Derbyshire KJ. 1993. Distribution of seagrasses, and their fish and penaeid prawn communities, in Cairns Harbour, a Tropical Estuary, Northern Queensland, Australia. Aust J Mar Freshw Res 44: 193-210. 
Cucio C, Engelen AH, Costa R, Muyzer G. 2016. Rhizosphere microbiomes of European seagrasses are selected by the plant, but are not species specific. Front Microbiol 7: 1-15.

den Hartog C. 1970. The Sea-grasses of the Word. North-Holland Publishing Co., Amsterdam.

DPKP Bontang. 2011. Rencana Strategis Tahun 2011-2016. Dinas Perikanan, Kelautan dan Pertanian (DPKP) Kota Bontang, Bontang. [Indonesian]

DPKP Bontang. 2015. Laporan Akhir Pendampingan Penyusunan Kajian Review Rencana Zonasi Pesisir, Laut dan Pulau-Pulau Kecil Pesisi Kota Bontang. Dinas Perikanan, Kelautan dan Pertanian (DPKP) Kota Bontang, Bontang. [Indonesian]

Dromard C.R, Vaslet A, Gautier F, Bouchon-Navaro Y, Harmelin-Vivien M, Bouchon C. 2017. Resource use by three juvenile scarids (Cryptotomus roseus, Scarus iseri, Sparisoma radians) in Caribbean seagrass beds. Aquat Bot 136: 1-8.

Duarte CM, Sintes T, Marba N. 2013. Assessing the $\mathrm{CO}_{2}$ capture potentia of seagrass restoration projects. Journal of Applied Ecology 50: 13411349.

English S, Wilkinson C, Baker V. 1994. Survey Manual For Tropica Marine Resources. Townville, Australia. ASEAN-Australia Marine Science. Australian Institute of Marine Science, Townsville. $\square \square$

Erftemeijer PLA, Allen GR. 1993. Fish fauna of seagrass beds in South Sulawesi, Indonesia. Rac West Aust Mus 16 (2): 277.

FAO. 2013. Fisheries and Aquaculture Department. FAO, Rome, Italy. http: //www.fao.org/fishery

Fishery Research Center [Balai Riset Perikanan Laut]. 2009. Ikan Hias Laut Indonesia. Balai Riset Perikanan Laut, Depok. [Indonesian]

Fonseca MS, Cahalan JA. 1992. A Preliminary Evaluation of Wave Attenuation by Four Species of Seagrass. Estuar Coast Shelf Sci 35 (6): $565-576$

Fortes DM. 1993. Taxonomy and Distribution of Seagrasses in the ASEAN Region. In Study No. 6 Seagrasses Resources in Southeast Asia. UNESCO, Jakarta.

Grech A, Miller KC, Erftemeijer P, Fonseca M, McKenzie L, Rasheed M, Taylor H, Coles R. 2012. A comparison of threats, vulnerabilities, and management approaches in global seagrass bioregion. Environ Res Lett 7: 1-8. DOI: 10.1088/1748-9326/7/2/024006.

Greening H, Janicki A. 2006. Toward reversal of eutrophic conditions in a subtropical estuary: Water quality and seagrass response to nitrogen loading reductions in Tampa Bay, Florida, USA. Environ Manag 38 (2): 163-178

Guannel G, Arkema K, Ruggiero P, Verutes G. 2016. The power of three: coral reefs, seagrasses and mangroves protect coastal regions and increase their resilience. PLoS ONE 11 (7): 1-22. DOI 10.1371/journal.pone.0158094.

Hantanirina JMO, Benbow S. 2013. Diversity and coverage of seagrass ecosystems in south-west Madagascar. Africa J Mar Sci 35 (2): 291297.

Hirst AJ, Longmore AR, Ball D, Cook PLM, Jenkins GP. 2016. Linking nitrogen sources utilized by seagrass in a temperate marine embayment to patterns of seagrass change during drought. Mar Ecol Prog Ser 549: 79-88.

Honda K, Nakamura Y, Nakaoka M, Uy WH, Forttes MD. Masahiro. 2013. Habitat use by fishes in coral reefs, seagrass beds and mangrove habitats in the Philippines. PLoS ONE 8(8): e65735. DOI: 10.1371/journal.pone.0065735.

Hughes AR, Williams SL, Duarte CM, Heek Jr. KN, Waycott M. 2009. Association of concern: declining seagrasses and threatened dependent species. Front Ecol Environ 7 (5): 242-246.

John BM, Shirlal KG, Rao S. 2015. Effect of artificial sea grass on wave attenuation-an experimental investigation. Internasional Conference on Water Resources, Coastal and Ocean Engineering (ICWRCOE 2015). Aquatic Procedia 4: 221-226.

Jones B, Shimlock M, Erdmann M, Allen G. 2011. Diving Indonesia Bird's Head Seascape. Saritaksu Edition, Bali.

Jones CM. 2014. Can we predict the future: juvenile finfish and their seagrass nurseries in the Chesapeake Bay. ICES J Mar Sci 71 (3): 681-688

Kuo J, McComb AJ. 1989. Seagrass Taxonomy, Structure and Development. In: Larkum AWD, McComb AJ, Shaphered SA (eds.) Aquatic Plant Studies 2: Biology of Seagrasses. Elsevier, Dordrecht. $\square \square$

Lepoint G, Michel LC, Parmentier E, Frédérich B. 2016. Trophic ecology of the seagrass-inhabiting footballer demoiselle Chrysiptera annulata
(Peters, 1855); comparison with three other reef-associated damselfishes. Belgian J Zool 146 (1): 21-32.

Lotuconsina H, Ambo-Rappe A, Nessa MN. 2012. The composition of species and structure of seagrass fish community in Tanjung TiramInner Ambon Bay. Jurnal Ilmu dan Teknologi Kelautan Tropis 4 (1): 35-46. [Indonesian]

Ludwig JA, Reynolds JF. Statistical Ecology: A Primer Methods and Computing. John Wiley \& Sons, New York.

Mazarrasa I, Marbà N, Lovelock CE, Serrano O, Lavery PS, Fourqurean JW, Kennedy H, Mateo MA, Krause-Jensen D, Steven ADL, Duarte CM. 2015. Seagrass meadows as a globally significant carbonate reservoir. Biogeosci Discuss 12: 4107-4138.

McCloskey RM, Unsworth RKF. 2015. Decreasing seagrass density negatively influences associated fauna. PeerJ 3: e1053. DOI: 10.7717 /peerj. 1053

Nadiarti, Riani E, Djuwita I, Budiharsono S, Purbayanto A, Asmus H. 2012. Challenging for seagrass management in Indonesia. J Coast Dev 15 (3): 234-242.

Olaniyan RF, 2015. Fishing Methods and their Implications for a sustainable environment. Fish Aquac J 6: 139. DOI: 10.4172/21503508.1000139

Oliveira RR des S, Macieira RM, Giarrizzo T. 2016. Ontogenetic shifts in shes between vegetatedis:epiand unvegetated tidepools: assessing the effect of physical structure on sh habitat selection. J Fish Biol 89: 959-976.

P3IK/P3O-LIPI. 2000. Penelitian Sumberdaya Kelautan di kawasan Pengembangan dan Pengelolaan Wilayah Laut Pantai Kalimantan Timur: Bidang Biologi, Proyek Pengembangan dan Penerapan Iptek Kelautan/Pusat Penelitian dan Pengembangan Oseanologi-Lembaga Ilmu Pengetahuan Indonesia, Jakarta. [Indonesian]

Phinrub W, Montien-Art B, Promya J, Suvarnaraksha A. 2014. Fish diversity and fish community in seagrass beds at Ban Pak Klong, Trang Province, Thailand. Intl J Fish Aquat Stud 2 (2): 197-201.

Rasheed MA, Unsworth RKF. 2011. Long-term climate-associated dynamics of a tropical seagrass meadow: implications for the future. Mar Ecol Prog Ser 422: 93-103.

Rudi E, Muchsin I. 2011. Ikan Karang Perairan Aceh dan sekitarnya. Lubuk Agung, Bandung. [Indonesian]

Shafer DJ, Kaldy JE, Gaeckle JL. 2014. Science and Management of the Introduced Seagrass Zostera japonica in North America. Environ Manag 53: 147-162.

Sharifuddin. 2012. Dunia Ikan. Gajah Mada University Press. Yogyakarta. [Indonesian]

Short FT, Polidoro B, Livingstone SR, Carpenter KE, Bandeira S, Bujang JS, Calumpong HP, Carruthers TJB, Coles RG, Dennison WC, Erftemeijer PLA, Fortes MD, Freeman AS, Jagtap TG, Kamal AHM, Kendrick GA, Kenworthy WJ, La Nafie YA, Nasution IM, Orth RJ, Prathep A, Sanciangco JC, van Tussenbroek B, Vergara SG, Waycott M, Zieman JC. 2011. Extinction risk assessment of the world's seagrass species. Biol Conserv 144: 1961-1971.

STATISTICA v.8.0. 2007. STATISTICA Vesion 8.0. StatSoft. Inc. 19842007. USA.

Suyatna I, Sidik AS, Almadi IF, Rizal S, Sukarti K. 2016. Fish community structure in high water temperature around Bontang Industrial Estate, East Kalimantan, Indonesia. Biodiversitas 17 (2): 558-564

ter Braak CJF, Verdonschot P.F.M. Canonical correspondence analysis and related multivariate methods in aquatic ecology. Aquatic Sciences 57/3: 255-289.

ter Braak CJF. 1987. The analysis of vegetation-environment relationships by canonical correspondence analysis. Vegetatio 69: 69-77.

Tomascik T, Mah AJ, Nontji A, Moosa MK. 1997. The Ecology of Indonesia. Part I and II. Periplus Editions (HK) Ltd., Singapore.

Unsworth RKF, Bell JJ, Smith DJ. 2007. Tidal fish connectivity of reef and seagrass habitats in the Indo-Pacific. J Mar Biol Assoc UK 87: 1287-1296.

Unsworth RKF, Collier CJ, Henderson GM, McKenzie LJ. 2012. Tropical seagrass meadows modify seawater carbon chemistry: implications for coral reefs impacted by ocean acidification. Environ Res Lett 7 : 024026. DOI: $10.1088 / 1748-9326 / 7 / 2 / 024026$.

Verhoef E. 2009. Tropical Fish. Rebo International, Netherland.

Verweij MC, Nagelkerken I, Hans I, Ruseler SM, Mason PRD. 2008. Seagrass nurseries contribute to coral reef fish populations. Limnol Oceanogr 53 (4): 1540-1547.

Waycott M, Duarte CM., Carruthers TJB, Orth RJ, Dennison WC, Olyarnik S, Calladine A, Fourqurean JW, Heck KL, Hughes Jr. AR, Kendrick GA, Kenworthy WJ, Short FT, Williams SL. 2009. 
Accelerating loss of seagrasses across the globe threatens coastal ecosystems. Proc Natl Acad Sci USA 106 (30): 12377-12381.

Weiß CH. 2007. StatSoft, Inc., Tulsa, OK.: Statistica, Version 8. StA 91: 339-341.
Wood E, Michael AW. 2011. Reef Fishes of South-East Asia. New Holland, London. 